\title{
CPK Increased, CTCAE
}

National Cancer Institute

\section{Source}

National Cancer Institute. CPK Increased, CT CAE. NCI Thesaurus. Code C143389.

A finding based on laboratory test results that indicate an increase in levels of creatine phosphokinase in a blood specimen. 